Document downloaded from:

http://hdl.handle.net/10251/103635

This paper must be cited as:

Contat-Rodrigo, L.; Pérez Fuster, C.; Lidon-Roger, JV.; Bonfiglio, A.; Garcia-Breijo, E. (2017). Screen-printed Organic Electrochemical Transistors for the detection of ascorbic acid in food. Organic Electronics. 45:89-96. doi:10.1016/j.orgel.2017.02.037

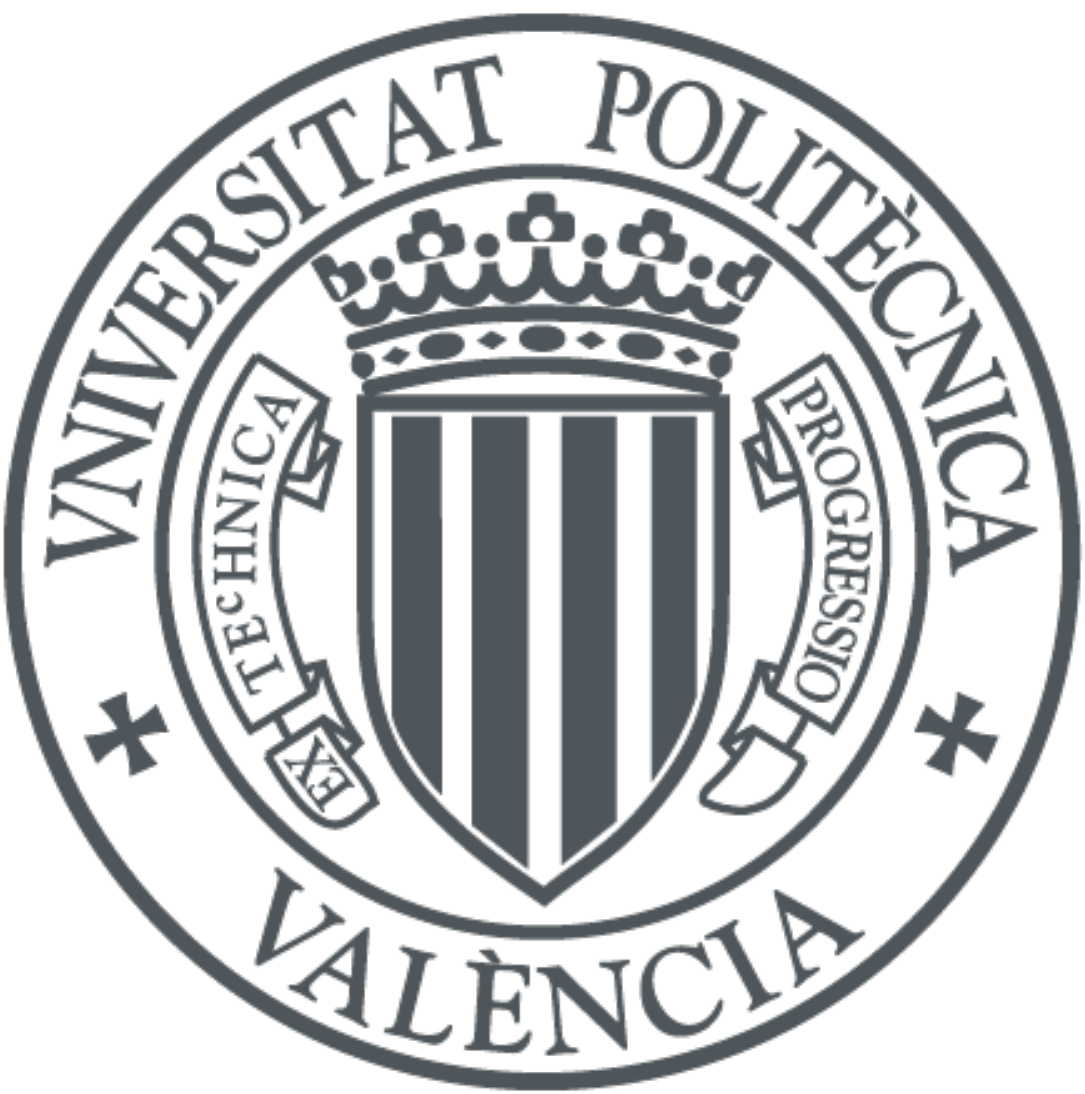

The final publication is available at

http://doi.org/10.1016/j.orgel.2017.02.037

Copyright Elsevier

Additional Information 


\section{Accepted Manuscript}

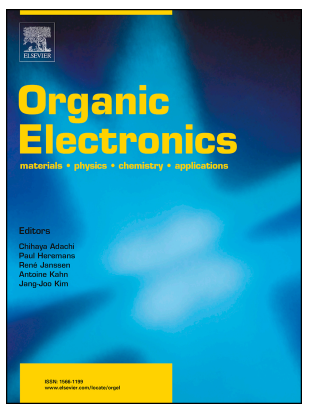

Screen-printed Organic Electrochemical Transistors for the detection of ascorbic acid in food

Laura Contat-Rodrigo, Clara Pérez-Fuster, José Vicente Lidón-Roger, Annalisa

Bonfiglio, Eduardo García-Breijo

PII: S1566-1199(17)30099-X

DOI: $\quad$ 10.1016/j.orgel.2017.02.037

Reference: ORGELE 3993

To appear in: Organic Electronics

Received Date: 10 January 2017

Revised Date: 23 February 2017

Accepted Date: 25 February 2017

Please cite this article as: L. Contat-Rodrigo, C. Pérez-Fuster, J.V. Lidón-Roger, A. Bonfiglio, E. GarcíaBreijo, Screen-printed Organic Electrochemical Transistors for the detection of ascorbic acid in food, Organic Electronics (2017), doi: 10.1016/j.orgel.2017.02.037.

This is a PDF file of an unedited manuscript that has been accepted for publication. As a service to our customers we are providing this early version of the manuscript. The manuscript will undergo copyediting, typesetting, and review of the resulting proof before it is published in its final form. Please note that during the production process errors may be discovered which could affect the content, and all legal disclaimers that apply to the journal pertain. 


\section{Screen-Printed Organic Electrochemical Transistors for the Detection of Ascorbic Acid in Food}

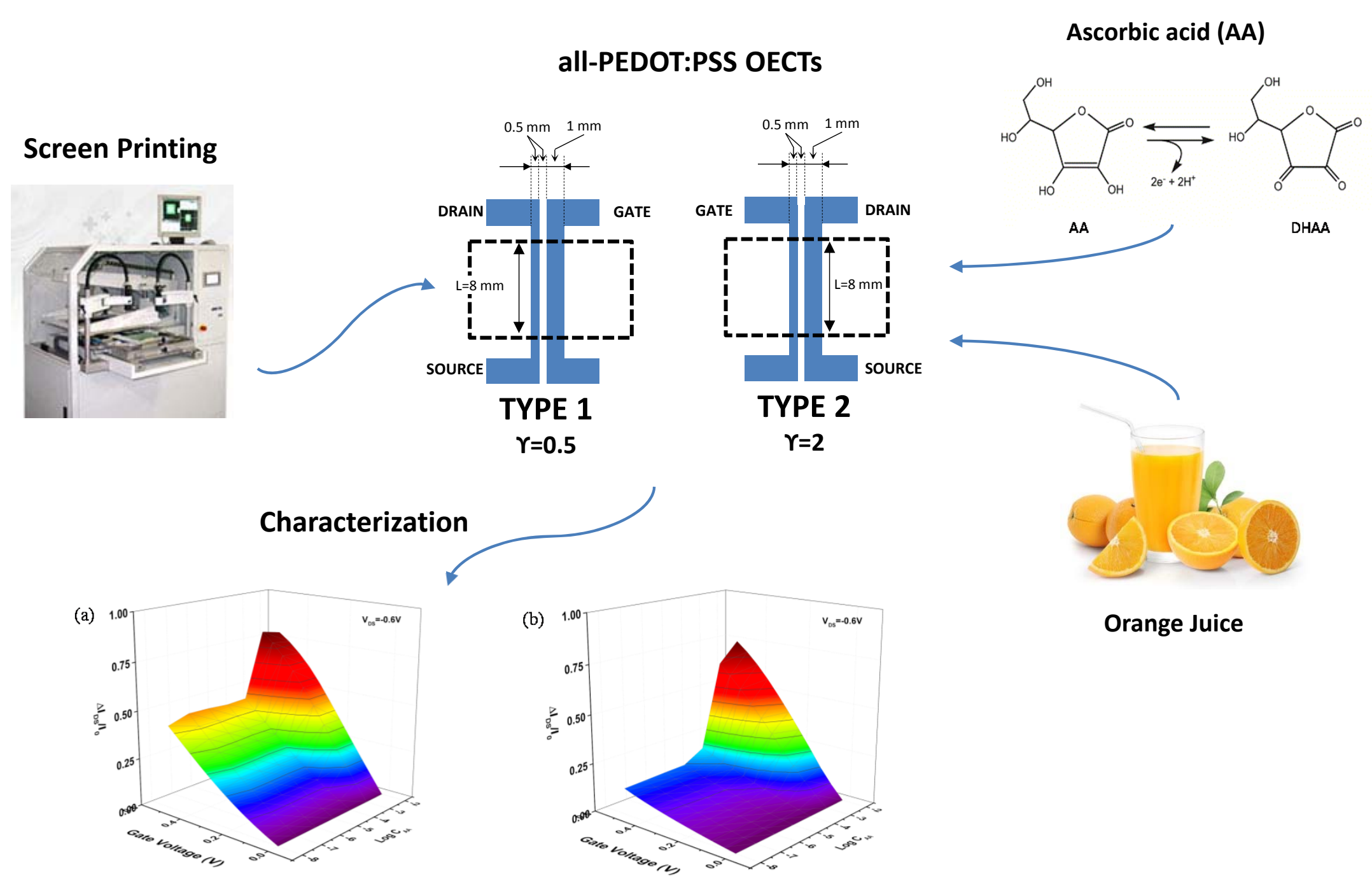




\title{
Screen-Printed Organic Electrochemical Transistors for the Detection of Ascorbic Acid in Food
}

\author{
Laura Contat-Rodrigo ${ }^{1}$, Clara Pérez-Fuster ${ }^{1}$, José Vicente Lidón-Roger ${ }^{1}$, Annalisa Bonfiglio ${ }^{2}$ \\ and Eduardo García-Breijo ${ }^{1, *}$
}

\author{
1 Group of Electronic Development and Printed Sensors (GEDPS), Instituto Universitario de \\ Reconocimiento Molecular y Desarrollo Tecnológico (IDM), Universitat Politècnica de València, \\ Camino de Vera s/n, 46022 Valencia, Spain \\ ${ }^{2}$ Dipartimento di Ingegneria Elettrica ed Elettronica. Università di Cagliari, Piazza d'Armi 09123, \\ Cagliari, Italy. \\ *e-mail: egarciab@eln.upv.es. Tel: +34 963877608
}

\begin{abstract}
Methods traditionally used for ascorbic acid (AA) detection in food are often expensive and complex, making them unsuitable for day-to-day determinations. In this work, we report on the use of all-PEDOT:PSS Organic Electrochemical Transistors (OECTs) for fast, simple and low-cost determination of AA in food. The performance of these OECTs was tested first with in lab-prepared solutions of AA with different concentrations. The effect of the geometry on the transistors performance for AA sensing was also investigated by comparing the response of two OECTs with different channel and gate areas ratio $(\gamma)$, in terms of current modulation, sensitivity, background signal and limit of detection (LOD). OECTs with smaller gate electrode than the channel (large $\gamma$ ) show the best performance for AA sensing: these devices display smaller background signal, higher sensitivity, larger modulation and better LOD value $(80 \mu \mathrm{M})$. Since the AA content in food rich in Vitamin C is in the $\mathrm{mM}$ range, these transistors can be considered sensitive enough for quantitatively monitoring AA in food. In order to demonstrate the reliability of the proposed sensors in real food samples, the response of these transistors was additionally measured in a commercial orange juice. The amount of AA obtained with the OECTs is in good agreement with that determined by HPLC and with values reported in the literature for orange juices. Furthermore, these OECTs can be considered promising candidates for the selective detection of AA in the presence of other interfering antioxidants.
\end{abstract}

\section{Keywords}

Organic Electrochemical Transistors; screen-printing; PEDOT:PSS; ascorbic acid

\section{Introduction}

Ascorbic acid (AA), commonly known as Vitamin C, is a naturally occurring hydrosoluble organic compound that can be found in many biological systems and food products, namely fresh vegetables and fruits. In aqueous solution, AA shows two deprotonation steps (with pKa values of 4.17 and 11.57) [1]. Therefore, at typical biological $\mathrm{pH}$ values, AA occurs as monodeprotonated ascorbate anion. AA is known for its reductive properties, being easily oxidized to dehydroascorbic acid (DHAA) (Figure 1). The oxidation of AA involves release of two electrons and two protons to produce DHAA [2]. Due to its strong antioxidant activity, AA is largely used as an antioxidant additive in food industry to prevent unwanted changes in color or flavor of food and drinks. Furthermore, the content of Vitamin $\mathrm{C}$ is used as an index of the health-related quality of fruits and vegetables [3]. Consequently, there is increasing interest in the development of analytical methods for the quantitative determination of AA in food. 


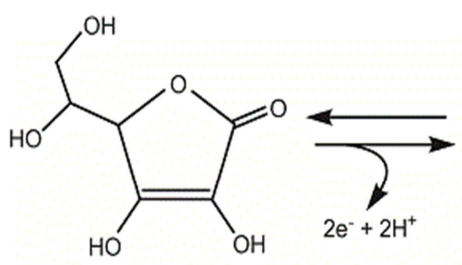

AA

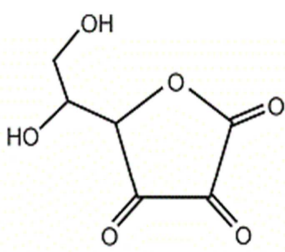

DHAA

Figure 1. Ascorbic acid (AA) and its two-electron oxidation product dehydroascorbic acid (DHAA) redox couple (redox potential $\mathrm{E}_{0}=+0.058 \mathrm{~V}$ ).

Traditional methods for the determination of AA in food include enzymatic techniques [4], liquid chromatography [5-7], spectrophotometry [8], chemiluminescence [9], capillary electrophoresis [10], and titration with an oxidant solution [11]. However, such detection methods often require expensive and complex instrumentation, making them unsuitable for day-to-day determinations. Therefore, the development of sensitive, simple, fast, and low cost techniques for the detection of AA is of great interest for food industry.

Electrochemical sensors can be a promising solution since they are based on the use of quick and easy procedures [2]. Among them, sensors based on organic semiconductor devices, like Organic Electrochemical Transistors (OECTs), can be regarded as a valid option for AA sensing. OECTs have attracted considerable interest in recent years for their application as chemical and biological sensors, due to their ability to operate in aqueous environments, easy fabrication, high sensitivity, and their simpler and cheaper readout electronics compared to conventional electrochemical sensors [12,13]. The essential components of an OECT are an organic semiconductor film, the channel (with source and drain electrodes), and an electrolyte bridging the channel and the gate electrode. As a convention, the source electrode is grounded and a voltage is applied to the drain electrode relative to ground. The operation of OECTs is based on the modulation of the channel current by electrochemical doping or de-doping from the electrolyte when gate voltages are applied.

One of the most widely used conducting polymers in these devices is poly(3,4ethylenedioxythiophene) doped with polysterene sulfonate (PEDOT:PSS) [14,15]. PEDOT:PSS is commercially available and can be readily patterned by conventional photolithography $[16,17]$, or by solution processing techniques like inkjet-printing $[18,19]$ and screen-printing [20-22]. In addition, it exhibits high conductivity, excellent thermal stability and good stability in a wide $\mathrm{pH}$ range. Upon the application of a positive-gate voltage in PEDOT:PSS-based OECTs, cations from the electrolyte migrate into the conducting polymer $[23,24]$. This in turn de-dopes PEDOT:PSS and leads to a decrease of the channel current. De-doping occurs by reduction of highly conducting PEDOT $^{+}$to less conducting $\mathrm{PEDOT}^{0}$, according to the following electrochemical reaction [25]:

$$
n\left(\text { PEDOT }^{+}: \text {PSS }^{-}\right)+M^{n+}+n e^{-} \leftrightarrows n P E D O T^{0}+M^{n+}: n P S S^{-}
$$

where $M^{n+}$ is a cation in the electrolyte, $n$ is the number of charge of the cation and $e^{-}$is an electron from the source electrode. When the gate voltage is removed, cations migrate back 
into the electrolyte, and the original conductivity of the organic semiconductor is restored. As a result of this working principle, these devices have been successfully employed as chemical and biological sensors [24,26]. In OECTs based on PEDOT:PSS, the gate electrode [27], the device architecture (ratio between the channel and gate areas, channel geometry) [28,29], and the materials properties play an important role in the sensor performance.

PEDOT:PSS-based OECTs can operate either as ion-to-electron converters or as electrochemical sensors [29]. In OECTs that are used as ion-to-electron converters, a transient ionic current is converted into a change in the drain current. The application of a positive gate voltage causes an ionic current in the electrolyte. Cations from the electrolyte migrate into the conducting polymer and de-dope it, leading to the decrease of the drain current. It has been demonstrated that for operation as ion-to-electron converters, the use of a gate electrode that is much larger than the channel or a non-polarizable gate electrode improves the sensor performance [29].

In OECTs that are used as electrochemical sensors, charge transfer reactions between a species in the electrolyte and the gate electrode change the potential of the electrolyte, inducing a change in the drain current. For operation as electrochemical sensors, OECTs with gate electrode smaller than the channel exhibit higher sensitivity [29]. All-PEDOT:PSS OECTs can be used as electrochemical sensors for the determination of redox active species (like adrenaline, dopamine and AA) by exploiting the ability of this polymer to electrocatalytically oxidize such molecules at the gate electrode [21,30,31]. In particular, PEDOT can directly oxidize AA according to the following reaction scheme:

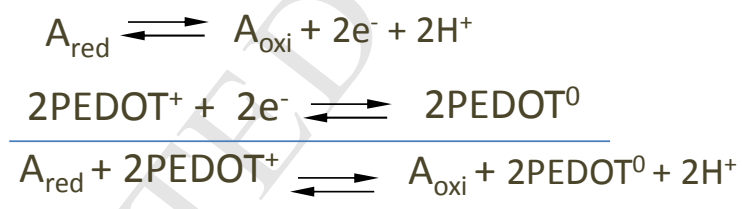

In addition, all-polymer sensors offer many advantages with respect to devices with metal gate electrode such as lower cost, easier fabrication and the possibility to be patterned in a flexible substrate.

In this communication, we report on the use of all-PEDOT:PSS OECTs as electrochemical sensors for the detection of AA in food. The performance of these OECTs was tested first with in lab-prepared solutions of AA with different concentrations. The effect of the geometry on the transistors performance for AA sensing was also investigated by comparing OECTs with different channel and gate areas ratio. Finally, the OECTs response was also measured in a commercial orange juice, in order to demonstrate the reliability of the proposed sensors in real food samples. 


\section{Materials and methods}

Preparation of PBS solution: A 0.1M Phosphate Buffered Saline (PBS) solution was prepared by dissolving $\mathrm{K}_{2} \mathrm{HPO}_{4}$ (Scharlau, Spain) and $\mathrm{KH}_{2} \mathrm{PO}_{4}$ (Scharlau, Spain) in Milli-Q water. The PBS solution had a $\mathrm{pH}$ of 4.8 as measured by $\mathrm{pH}$ meter.

Preparation of ascorbic acid solutions: Solutions were prepared by dissolving AA (Scharlau, Spain) in PBS solution with different molar concentrations $\left(10^{-8} \mathrm{M}, 10^{-7} \mathrm{M}, 10^{-6} \mathrm{M}\right.$, $10^{-5} \mathrm{M}, 10^{-4} \mathrm{M}, 10^{-3} \mathrm{M}$ and $\left.10^{-2} \mathrm{M}\right)$.

Orange juice: Commercial ready-to-drink orange juice (Hacendado, Spain) purchased from a local supermarket was used as a model real sample. The selected product contains, according to the label, $48 \mathrm{mg} / 100 \mathrm{ml}$ of Vitamin C. The juice was twice diluted with the PBS solution and used without further probe preparation.

Transistor Fabrication: Commercially available PEDOT:PSS C2100629D1 (Gwent, UK) with 500-700 $\Omega /$ sq was used as the active material for the fabrication of the OECTs. The channel and the gate were obtained by screen-printing PEDOT:PSS over a polyester film. Screen-printing was performed with PET 1500 165/420-34W/32 $\mu$ m mesh screen (Sefar, USA) and Dirasol 132 UV film (Fujifilm, USA). The final screen thickness was $55 \mu \mathrm{m}$. UV light was used to transfer the OECT pattern to the screen. The PEDOT:PSS ink was re-dispersed for 1 hour at $1000 \mathrm{rpm}$ before printing. Printing was carried out using an Aurel 900 screen-printer with $75^{\circ}$ shore hardness squeegee, 1 bar force and $0.2 \mathrm{~m} / \mathrm{s}$. Transparent and flexible Melinex ST506 polyester (DuPont Teijin Films, UK) of $175 \mu \mathrm{m}$ thickness was used as substrate, previously cleaned with acetone, ethanol and deionized water. Finally, the OECTs were cured in an air oven at $100^{\circ} \mathrm{C}$ for 10 minutes.

Electrical Measurements: All measurements were performed using a Keithley 4200 semiconductor characterization system.

High Performance Liquid Chromatography (HPLC): A LaChrom Elite system (Hitachi, Japan) equipped with an L-2400 UV detector, an L-2130 pump and an L-2200 auto sampler was used for HPLC analysis. To determine AA in the orange juice [32], $1 \mathrm{ml}$ sample was extracted with $9 \mathrm{ml} 0.1 \%$ oxalic acid for $3 \mathrm{~min}$ and immediately filtered before injection. The separation was carried out on an Ultrabase- $\mathrm{C}_{18}$ column $(250 \mathrm{~mm} \times 4.6 \mathrm{~mm}$ ) using $0.1 \%$ oxalic acid as the mobile phase at a flow rate of $1 \mathrm{ml} / \mathrm{min}$. Samples were injected three times in HPLC with a $20 \mu \mathrm{l}$ injection volume. The AA peak was detected at $243 \mathrm{~nm}$ and at $25^{\circ} \mathrm{C}$. The analysis was carried out by EZChrom Elite software (Agillent Technologies, USA).

Determination of the antioxidant activity: The antioxidant activity of the orange juice was assessed by means of the ABTS method for total antioxidant activity [33]. This technique measures the relative ability of antioxidant substances to scavenge the 2.2'azinobis(3-ethylbenzothiazoline-6-sulfonate) radical cation $\left(\mathrm{ABTS}^{\bullet+}\right)$. ABTS ${ }^{\bullet+}$ was prepared by mixing an ABTS stock solution $(15 \mathrm{mM})$ with potassium persulfate $(4.8 \mathrm{mM})$. The mixture (1:1) was allowed to stand in the dark at room temperature for $12 \mathrm{~h}$ before use. The working solution was prepared by dissolving $\mathrm{ABTS}^{\bullet+}$ radicalized solution in ethanol to an 
absorbance of $1.1 \pm 0.002$ at $\lambda=734 \mathrm{~nm}$. The spectrophotometric assay was performed by mixing $2850 \mu \mathrm{l}$ of the $\mathrm{ABTS}^{\bullet+}$ diluted solution with $150 \mu \mathrm{l}$ of the sample. Discoloration following the sample addition was determined by measuring the decrease of absorbance at $734 \mathrm{~nm}$ for $30 \mathrm{~min}$ at $20^{\circ} \mathrm{C}$. Appropriate solvent blanks were run for correction of the absorbance at each time.

\section{Results}

The screen-printed OECTs are composed of two parallel rectangular PEDOT:PSS stripes that act as channel and gate (Figure 2). OECTs with two types of geometries were designed, by modifying the ratio between the channel and gate areas $\gamma=A_{c h} / A_{g}$ (where $A_{c h}$ is the channel area and $\mathrm{A}_{\mathrm{g}}$ is the gate area) (Table 1). Both OECTs are asymmetric devices with $\gamma=0.5$ and $\gamma=2$, respectively. The electrolyte is confined by a polydimethylsiloxane (PDMS) well $(1.7 \times 1.1 \times 1 \mathrm{~cm})$ in a small area (dashed line in Figure 2$)$ that partly covers the channel and the gate.
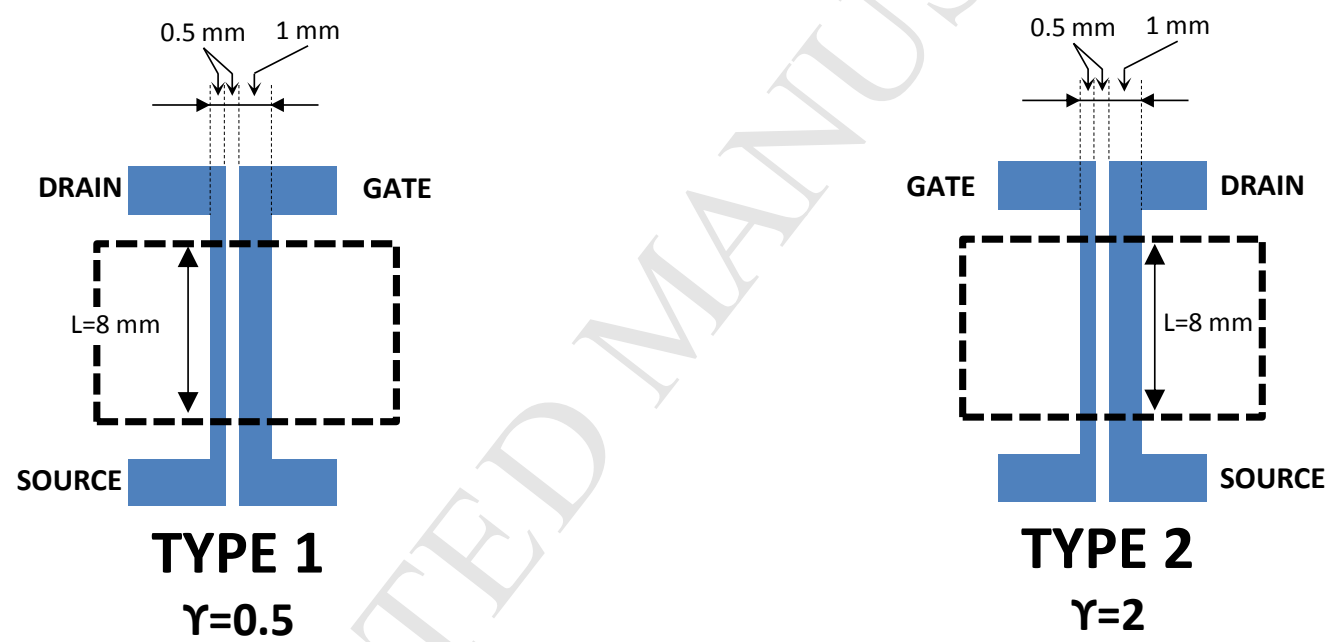

Figure 2. Schematic representation of the devices layout with different geometry.

Table 1. Geometric characteristics of the two types of OECTs.

\begin{tabular}{lcc}
\hline & Type 1 & Type 2 \\
\hline Channel width $\left(\mathrm{W}_{\mathrm{ch}}\right)(\mathrm{mm})$ & 0.5 & 1 \\
Channel length $\left(\mathrm{Lch}_{\mathrm{ch}}\right)(\mathrm{mm})$ & 8 & 8 \\
$\mathrm{~W}_{\mathrm{ch}} / \mathrm{Lch}$ & 0.0625 & 0.125 \\
Channel area $\left(\mathrm{A}_{\mathrm{ch}}\right)\left(\mathrm{mm}^{2}\right)$ & 4 & 8 \\
Gate area $\left(\mathrm{Ag}_{\mathrm{g}}\right)\left(\mathrm{mm}^{2}\right)$ & 8 & 4 \\
$\gamma=\mathrm{A}_{\mathrm{ch}} / \mathrm{A}_{\mathrm{g}}$ & 0.5 & 2 \\
\hline
\end{tabular}




\subsection{Electrical characterization of the OECTs in PBS solution}

These screen-printed OECTs were initially characterized in PBS solution. The output characteristics, the transfer characteristics and the current modulation of these two OECTs were determined and compared. The output characteristics were obtained by measuring the drain current $\left(\mathrm{I}_{\mathrm{DS}}\right)$ as a function of the drain voltage $\left(\mathrm{V}_{\mathrm{DS}}\right)$ for different gate voltages $\left(\mathrm{V}_{\mathrm{GS}}\right)$. Results show that the geometry has large influence on the output characteristics of these transistors (Figure 3). OECTs with small area ratio $(\gamma=0.5)$ exhibit strong modulation of the drain current, while the modulation of this current is weaker for transistors with large area ratio $(\gamma=2)[29]$.
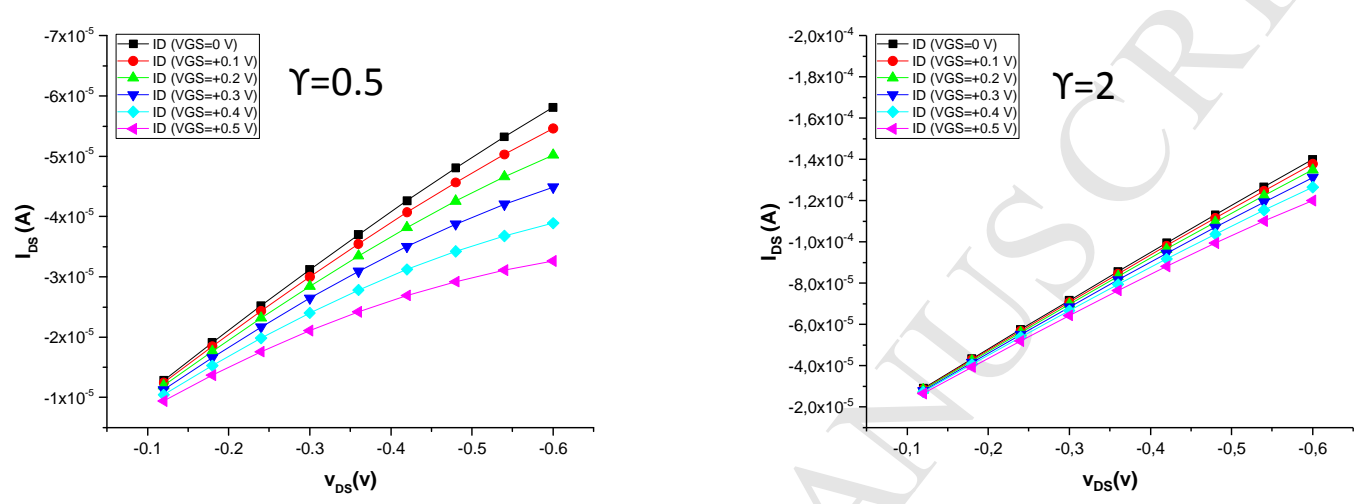

Figure 3. Output characteristics of the OECTs with different values of $\gamma$.

Concerning the transfer characteristics of these OECTs, these were obtained plotting $\mathrm{I}_{\mathrm{DS}}$ (normalized to its maximum value) vs $\mathrm{V}_{\mathrm{GS}}$ from $0 \mathrm{~V}$ to $0.5 \mathrm{~V}$, under constant drain voltage (Figure 4a). Results confirm that OECTs with larger gate electrode than the channel (small $\gamma$ ) display higher modulation of the drain current. In contrast, OECTs with smaller gate electrode than the channel (large $\gamma$ ) show little modulation of the drain current and remain in the on state for a wider range of the applied $\mathrm{V}_{\mathrm{GS}}$ [29].
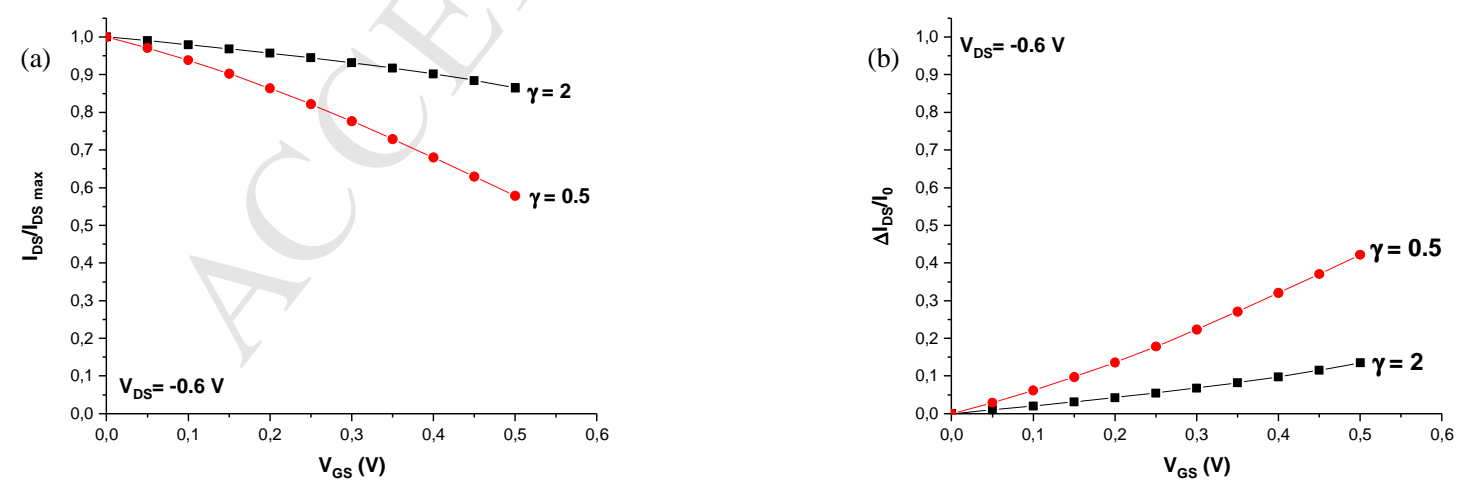

Figure 4. Curves of the OECTs with different values of $\gamma\left(V_{D S}=-0.6 V\right)$ : (a) normalized $I_{D S}$ vs $V_{G S}$ and (b) current modulation $\Delta \mathrm{I}_{\mathrm{DS}} / \mathrm{I}_{0}$ vs $\mathrm{V}_{\mathrm{GS}}$.

Finally, the current modulation $\left(\Delta \mathrm{I}_{\mathrm{DS}} / \mathrm{I}_{0}\right)$ of these OECTs was obtained as a function of the gate voltage for different values of $\gamma$ : 


$$
\Delta I_{D S} / I_{0}=\left|I_{D S}-I_{0}\right| / I_{0}
$$

where $\mathrm{I}_{\mathrm{DS}}$ is the off current $\left(\mathrm{V}_{\mathrm{GS}} \neq 0\right)$ and $\mathrm{I}_{0}$ is the on current $\left(\mathrm{V}_{\mathrm{GS}}=0\right)$. Current modulation strongly decreases with increasing the area ratio ( Figure 4b). OECTs type 1 with small $\gamma$ exhibit strong modulation throughout all the investigated $\mathrm{V}_{\mathrm{GS}}$ range, while OECTs type 2 with large $\gamma$ exhibit little modulation.

This is in good agreement with the idea that two regimes can be identified in OECTs operating like electrochemical sensors [34]: a non-Faradaic regime (in the absence of analyte) where the polymer doping is coupled to the charging of an ionic double layer at the gate electrode, and a Faradaic regime (in the presence of analyte) where doping/dedoping is coupled to a redox reaction at the gate electrode.

Here, in the absence of AA, with just PBS in the electrolyte reservoir, there is no charge transfer between the electrolyte and the gate electrode, and OECTs operate in the nonFaradaic regime. The electrolyte potential $\left(\mathrm{V}_{\mathrm{sol}}\right)$, in this case, is determined by the capacitances associated with double layer formation at the gate and the channel [35]:

$$
V_{s o l}=V_{G S} /\left(1+\left(c_{c h} A_{c h} / c_{g} A_{g}\right)\right) \approx V_{G S} /(1+\gamma)
$$

where $c_{c h}$ and $c_{g}$ are the channel and gate capacitances per unit area, respectively. If it is assumed that these capacitances are similar, the electrolyte potential only depends on the ratio between the channel and gate areas $(\gamma)$ [29]. In OECTs with larger gate electrode than the channel $(\gamma<1)$, the electrolyte is nearly at the same potential as the gate electrode. This leads to a large potential drop at the electrolyte/channel interface, resulting in a strong modulation of the drain current. In contrast, in OECTs with smaller gate electrode than the channel $(\gamma>1)$, the electrolyte is nearly at the same potential than the channel. This results in a small potential drop at the electrolyte/channel interface, which in turn leads to a weak modulation of the drain current. Therefore, for operation in the non-Faradaic regime, the current modulation of these OECTs decreases strongly with $\gamma$.

\subsection{OECTs response in ascorbic acid solutions}

\section{Effect of the geometry}

Next, the response of the OECTs was tested in AA solutions of concentrations ranging from $10^{-8} \mathrm{M}$ to $10^{-2} \mathrm{M}$. The gate-source current $\left(\mathrm{I}_{\mathrm{GS}}\right)$ was initially measured as a function of $\mathrm{V}_{\mathrm{GS}}$ and $\mathrm{AA}$ concentration for the two types of OECTs. $\mathrm{I}_{\mathrm{GS}}$ can be used as a fingerprint of the operation regime of the transistors (non-Faradaic or Faradaic), since this is due to capacitative effects and/or charge transfer reactions at the gate electrode [27]. Results show the presence of a large $\mathrm{I}_{\mathrm{GS}}$ in both devices for high concentrations of $\mathrm{AA}\left(\geq 10^{-4} \mathrm{M}\right)$, consistent with the presence of Faradaic processes at the gate electrode (Figure 5). In 
contrast, both transistors show a small $\mathrm{I}_{\mathrm{GS}}$ at low concentrations of AA $\left(\leq 10^{-5} \mathrm{M}\right)$, indicative of a non-Faradaic operation regime.

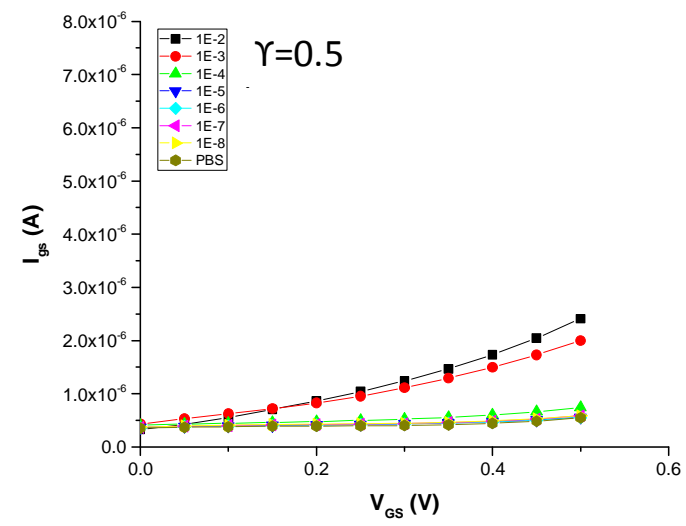

Figure 5. $\mathrm{I}_{\mathrm{GS}}$ as a function of $\mathrm{V}_{\mathrm{GS}}$ and $\mathrm{AA}$ concentration for the OECTs with different values of $\gamma\left(\mathrm{V}_{\mathrm{DS}}=-0.6 \mathrm{~V}\right)$.

The current modulation $\left(\Delta \mathrm{I}_{\mathrm{DS}} / \mathrm{I}_{0}\right)$ was obtained as a function of AA concentration for various values of $V_{G S}$, where $I_{D S}$ is considered at zero concentration (no analyte) and at concentration of interest. This normalization facilitates comparison between the transistors with different geometry. The three-dimensional plots for the OECTs with different $\gamma$ are shown in Figure 6. The operation of these two OECTs yields a response that is dependent on AA concentration and $\mathrm{V}_{\mathrm{GS}}$, confirming that these devices can be used as AA sensors. In particular, the drain current increases with concentration, being this change larger as $\mathrm{V}_{\mathrm{GS}}$ increases [27,28,35]. These changes are, however, more significant for OECTs with large $\gamma$ (type 2). These results indicate that for detection of AA, OECTs with smaller gate electrode than the channel display stronger current modulation [28].

Figure 6. Normalized response of $I_{D S}$ as a function of the applied gate voltage and AA concentration for OECTs: (a) type 1 and (b) type $2,\left(\mathrm{~V}_{\mathrm{DS}}=-0.6 \mathrm{~V}\right)$.

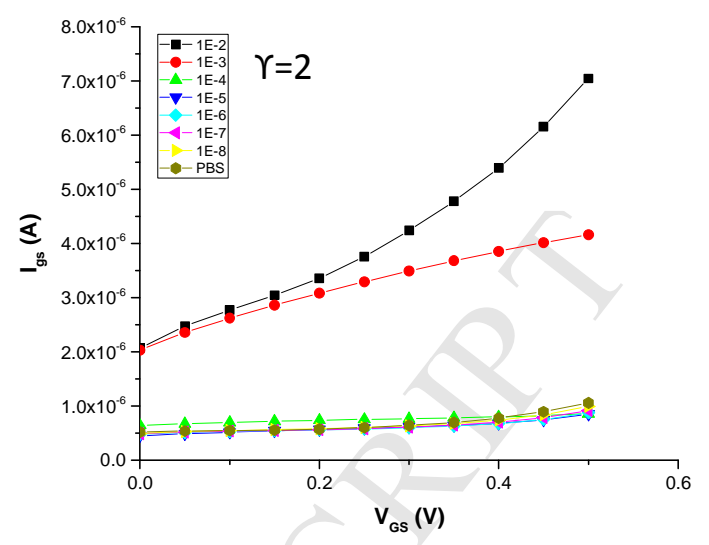

(a)

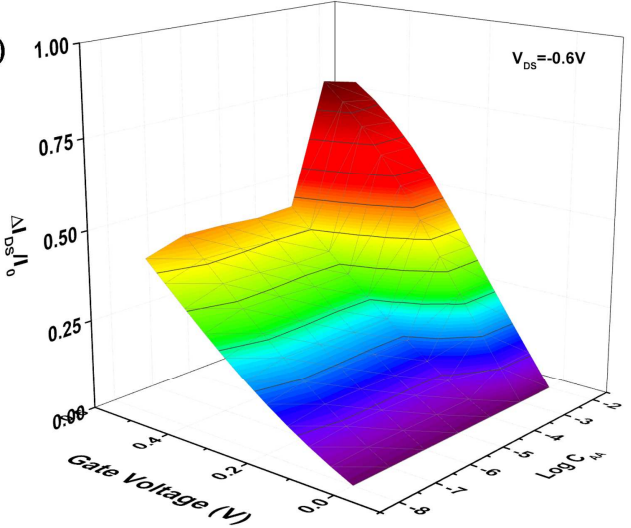

(b)

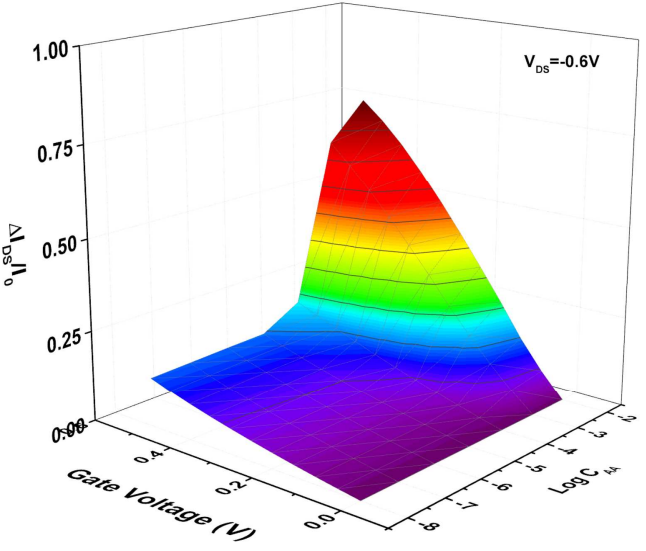


The influence of the ratio of the channel to gate areas in the response of these OECTs is highlighted by comparing the current modulation of the transistors with different values of $\gamma$, in AA solutions of various concentrations, at fixed $V_{\mathrm{GS}}$ (Figure 7). Results yield typical Sshaped curves characteristic of sensors, consisting of three distinct regimes [28]. First, for low concentrations ([AA] $\leq 10^{-5} \mathrm{M}$ ), a background $\Delta \mathrm{I}_{\mathrm{DS}} / \mathrm{I}_{0}$ is obtained. This background signal is independent on concentration and is of non-Faradaic origin, as previously shown in Figure 5. Then, $\Delta \mathrm{I}_{\mathrm{DS}} / \mathrm{I}_{0}$ increases with concentration $\left(10^{-5} \mathrm{M} \leq[\mathrm{AA}] \leq 10^{-2} \mathrm{M}\right)$. The current modulation is therefore enhanced in the presence of AA due to the Faradaic contribution that exists in this concentration range, as seen in Figure 5. For high concentrations ( $\left.[A A] \geq 10^{-2} M\right), \Delta I_{D S} / I_{0}$ tends to saturation. Saturation occurs when the electrolyte potential becomes similar to the applied gate voltage, and further addition of analyte does not result in further de-doping of the channel [28].

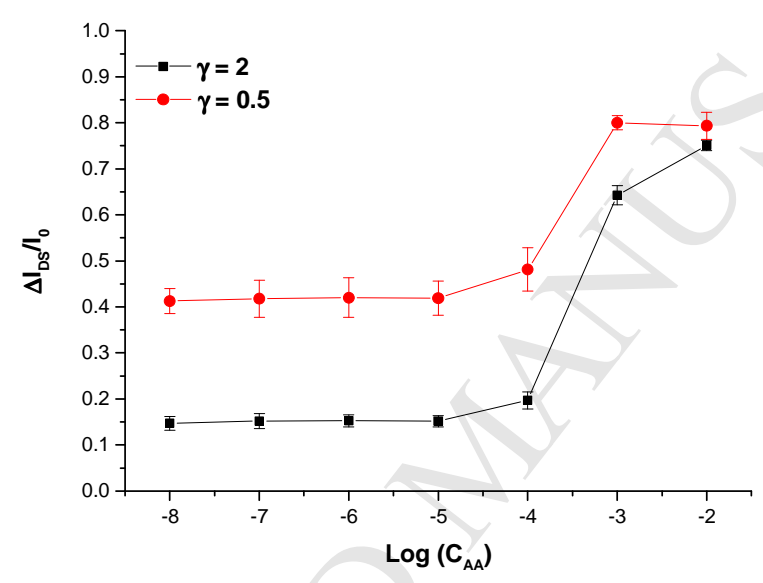

Figure 7. Response of the OECTs with different values of $\gamma$ as a function of AA concentration for $\mathrm{V}_{\mathrm{DS}}=-0.6 \mathrm{~V}$ and $\mathrm{V}_{\mathrm{GS}}=+0.5 \mathrm{~V}$.

These results demonstrate that $\gamma$ has a pronounced effect on the background signal and sensitivity (slope of the normalized response vs AA concentration) of these devices. The background value decreases with $\gamma$, whereas sensitivity increases with $\gamma$ (OECTs type 1 and type 2 show a sensitivity of $15.4 \mu \mathrm{A} \cdot \mathrm{dec}^{-1}$ and $61.2 \mu \mathrm{A} \cdot \mathrm{dec}^{-1}$, respectively). This means that transistors with smaller gate electrode than the channel (large $\gamma$ ) display small background signal and high sensitivity. In contrast, the saturation and detection range seem to be rather independent of $\gamma$. Saturation of $\Delta \mathrm{I}_{\mathrm{DS}} / \mathrm{I}_{0}$ seems to be at the same value and for the same AA concentration, regardless the value of $\gamma$. The minimum and maximum detectable concentrations are about $10^{-5} \mathrm{M}$ and $10^{-2} \mathrm{M}$, respectively, for both devices. These results are in good agreement with those reported by Cicoira et al. for PEDOT:PSS-based OECTs used for the detection of hydrogen peroxide via electrochemical oxidation at a Pt gate electrode [28].

In the presence of AA, the OECT operates in the Faradaic regime, since introduction of $\mathrm{AA}$ in the electrolyte results in charge transfer to the gate electrode according to the following reaction:

$$
A A_{\text {red }} \leftrightarrow A A_{o x}+2 H^{+}+2 e^{-}
$$


This transfer of electrons between the electrolyte and the gate electrode raises the electrolyte potential $\left(\mathrm{V}_{\text {sol }}\right)$ by a value described by the Nernst equation [34]:

$$
V_{\text {sol }}=\left[V_{G S} /(1+\gamma)\right]+(k T / 2 e) \ln [A A]+\text { constant }
$$

where $\mathrm{k}$ is the Boltzmann constant, $\mathrm{T}$ is the temperature, $\mathrm{e}$ is the fundamental charge, $\mathrm{c}$ is the concentration of AA, and the constant contains details such as the formal potential. Therefore, the addition of AA results in the decrease in the potential drop at the gate/electrolyte interface, which leads to the subsequent increase in the potential drop at the electrolyte/channel interface, since the gate is held at constant voltage. In OECTs with smaller gate electrode than the channel (large $\gamma$ ), a small $\mathrm{V}_{\text {sol }}$ is favored in the absence of AA (as confirmed previously by the characterization of these devices in PBS solution). Consequently, in OECTs with large $\gamma$, the modulation caused by the addition of AA will be proportionally larger than in OECTs with larger gate electrode than the channel (small $\gamma$ ). This can explain the dependence of the sensitivity and current modulation on $\gamma$ observed for these transistors: OECTs with large $\gamma$ display higher sensitivity and better modulation for AA sensing. These observations are also in good agreement with the findings of Cicoira et al. for $\mathrm{H}_{2} \mathrm{O}_{2}$ sensors [28].

\section{Effect of AA concentration on the OECTs response}

Since OECTs with large $\gamma$ (type 2) have shown the best performance for AA sensing in terms of sensitivity and current modulation, these transistors were chosen to further analyze the effect of AA concentration on their response. Although not shown here, similar results were obtained for OECTs type 1.

The redox reaction described above that occurs when AA is added to the electrolyte, also affects the source-drain current. This is illustrated in Figure 8a, which displays the transfer characteristics of OECTs type 2 for different AA concentrations. In all the investigated AA concentration range, $\mathrm{I}_{\mathrm{DS}}$ decreases with increasing $\mathrm{V}_{\mathrm{GS}}$ due to de-doping of PEDOT:PSS in the channel. However, the presence of AA clearly affects the transfer characteristics. With increasing AA concentration, the current modulation increases. In particular, modulation is enhanced for high AA concentrations ( $[\mathrm{AA}] \geq 10^{-4} \mathrm{M}$ ) due to the Faradaic contribution that exists in this concentration range. These observations are consistent with Figures 5 and 7.

The gate voltage can be scaled to yield a universal curve according to [35]:

$$
V_{G S}^{e f f}=V_{G S}+V_{o f f s e t}
$$

$\mathrm{V}_{\mathrm{GS}}^{\mathrm{eff}}$ is the effective gate voltage, that is defined as the equivalent voltage that needs to be applied in the absence of Faradaic effects at the gate electrode to obtain the same $I_{D S}$. $V_{\text {offset }}$ is an offset voltage that depends on AA concentration. It describes the Faradaic contribution to $\mathrm{V}_{\mathrm{GS}}^{\mathrm{eff}}$ and originates from the shift in the chemical potential described by the Nernst equation (Eq. 6):

$$
V_{\text {offset }}=(1+\gamma)(k T / 2 e) \ln [A A]+\text { constant }
$$


This universal curve agrees with the typical transfer characteristics of a depletion mode transistor, showing the monotonic decrease of $\mathrm{I}_{\mathrm{DS}}$ with increasing the gate voltage (Figure $8 b)$.
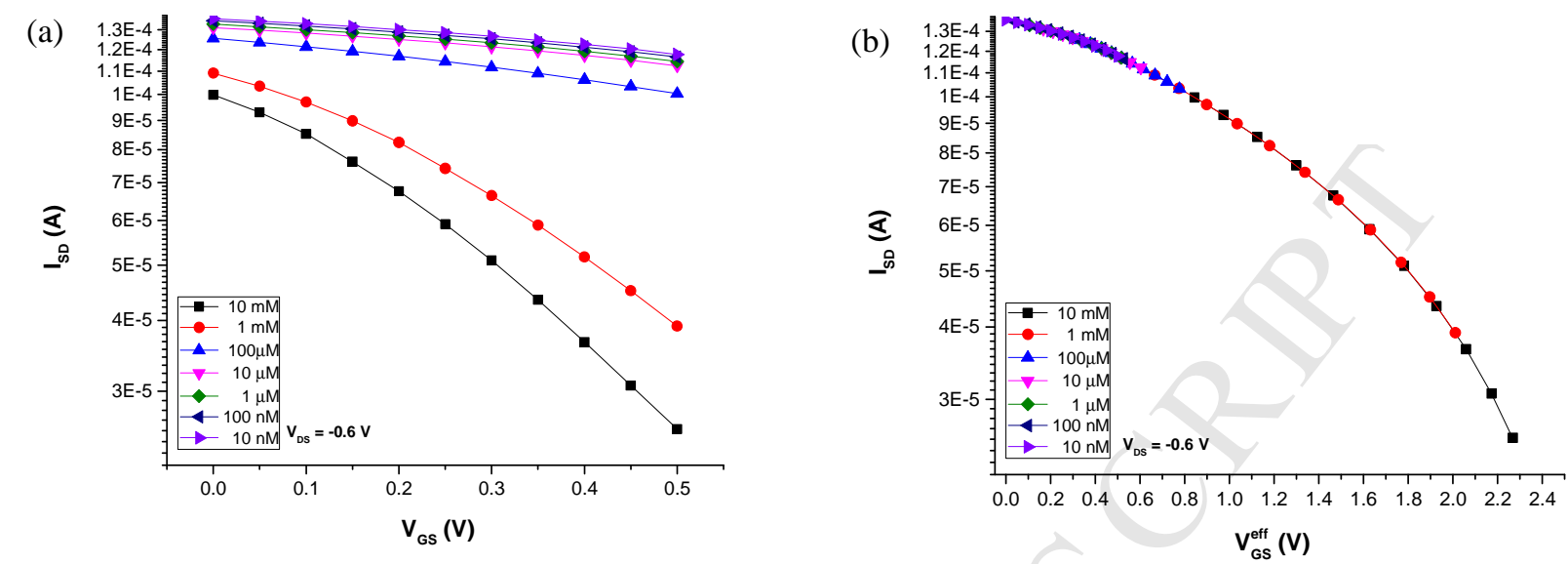

Figure 8. OECTs type $2(\gamma=2)$ : (a) $\mathrm{I}_{\mathrm{DS}}$ as a function of applied $\mathrm{V}_{\mathrm{GS}}$ for a fixed drain voltage $\left(\mathrm{V}_{\mathrm{DS}}=-\right.$ $0.6 \mathrm{~V}$ ) and various AA concentrations and (b) $\mathrm{I}_{\mathrm{DS}}$ as a function of effective gate voltage.

The effect of AA concentration on the transistors response is highlighted in Figure 9, in which $\mathrm{V}_{\text {offset }}$ of OECTs type 2 is plotted as a function of AA concentration. The gating of the transistors is clearly affected by the presence of AA. A logarithmic behavior (reminiscent of the Nernst equation) is observed for AA content in the range $10^{-5} \mathrm{M}-10^{-2} \mathrm{M}$, consistent with the charge transfer reaction that occurs at the gate electrode for these concentrations (Figure $5)$.

The limit of detection (LOD) of these OECTs was obtained to evaluate their performance in AA solutions. LOD was calculated as the concentration that leads to a signal three times higher than experimental noise:

$$
L O D=3 S b l / a
$$

where ( $\mathrm{Sbl}$ ) is evaluated as the standard deviation of the blank signal (PBS) and a is the slope obtained by linear fit. The LOD values estimated for OECTs type 1 and type 2 are $107.7 \mu \mathrm{M}$ and $80.1 \mu \mathrm{M}$, respectively. This confirms that OECTs with large $\gamma$ show the best performance for AA sensing, also in terms of LOD. These LOD values are, however, somewhat higher than that reported recently by Gualandi et al. for another all-PEDOT:PSS OECT for AA sensing [30]. The lower LOD obtained by these authors can be attributed to the different geometry of their proposed OECT (larger $\gamma$ and lower $\mathrm{W}_{\mathrm{ch}} / \mathrm{L}_{\mathrm{ch}}$ ratio). Taking into account that AA content in food rich in Vitamin $\mathrm{C}$ does not exceed the $\mathrm{mM}$ range [36], these transistors can be considered sensitive enough for quantitatively monitoring AA in food. 


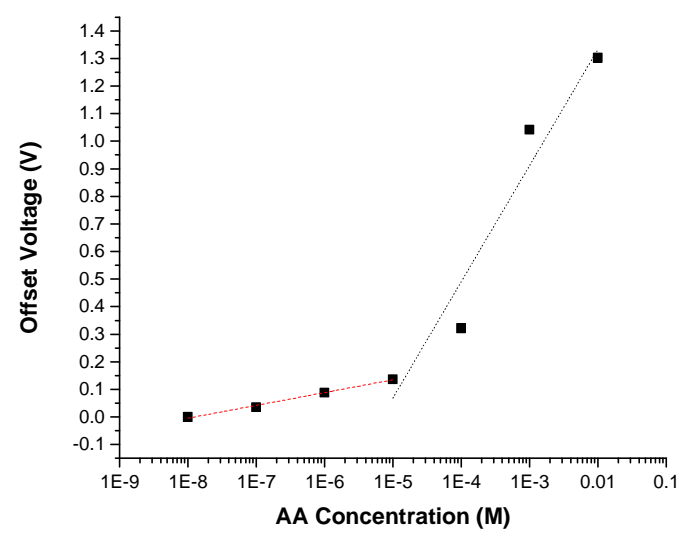

Figure 9. Dependence of the offset voltage on AA concentration for OECTs type 2.

\subsection{OECTs response in orange juice}

Finally, the OECTs were used to measure AA in commercial orange juice, to assess their response in real food samples. AA determination in orange juice was performed with OECTs type 2 with large $\gamma$, which have shown better sensitivity, current modulation and LOD in the previous tests with in-lab prepared AA solutions. The change in $\mathrm{I}_{\mathrm{DS}}$ was used to determine the AA concentration in the orange juice, which was found to be equal to 52.80 $\mathrm{mg} / 100 \mathrm{ml}$. This value is in good agreement with the amount of AA in the juice obtained by HPLC, which was equal to $56.71 \mathrm{mg} / 100 \mathrm{ml}$, and with that reported by Rapisarda et al. for fresh "Valencia Late" orange juice $(51.37 \mathrm{mg} / 100 \mathrm{ml})$ [37].

The antioxidant activity is an overall measure of the different antioxidant compounds that are present in a sample. In orange juices, these include AA but also carotenoids and, especially, phenolics (like flavonoids and phenolic acids), among others. The antioxidant activity of the orange juice was evaluated using the ABTS method. An antioxidant activity of $7.79 \mathrm{mM}$ was obtained, which is equivalent to $130.3 \mathrm{mg} / 100 \mathrm{ml}$, and is in good agreement with that reported by Rapisarda et al. (7.4 mM) [37]. Comparison of the antioxidant activity and the amount of AA in the orange juice confirms that, besides AA, this juice contains other antioxidant compounds. Furthermore, since the AA content measured with the OECTs has been found to be in good agreement with that determined by HPLC and with values reported in the literature, this suggests that these transistors can be considered promising candidates for selective AA detection. This preliminary conclusion should be however confirmed by performing additional cross experiments with interfering antioxidants present in orange juice.

\section{Conclusions}

The use of all-PEDOT:PSS OECTs as electrochemical sensors for the determination of AA content in food has been assessed. OECTs were prepared by screen-printing the organic semiconductor over a flexible substrate. The performance of these OECTs was first tested with in lab-prepared solutions of AA with different concentrations ranging from $10^{-8} \mathrm{M}$ 
to $10^{-2} \mathrm{M}$. Measurements of the gate-source current indicate that for low AA concentrations (up to $10^{-5} \mathrm{M}$ ), these OECTs operate in the non-Faradaic regime, whereas for high AA concentrations (up to $10^{-2} \mathrm{M}$ ), Faradaic processes occur at the gate electrode, resulting from the ability of PEDOT:PSS to electrocatalytically oxidize AA. The Faradaic contribution in this concentration range greatly enhances modulation of the drain current and the gating of the transistors.

The effect of the geometry on the performance of these devices for AA sensing was additionally investigated by comparing two OECTs with different channel and gate areas ratio $(\gamma)$, in terms of current modulation, sensitivity and background signal. It was found that the response to AA is improved in OECTs with smaller gate electrode than the channel (large $\gamma$ ), which display smaller background signal that results in higher sensitivity and larger modulation by the addition of AA to the electrolyte. OECTs with large $\gamma$ also display better LOD value $(80.1 \mu \mathrm{M})$, confirming that this geometry shows the best performance for AA sensing. Since the AA content in food rich in Vitamin $C$ is in the $\mathrm{mM}$ range, these transistors can be considered sensitive enough for quantitatively monitoring AA in food.

To demonstrate the reliability of the proposed sensors in real food samples, the OECTs were used to measure the AA content in a commercial orange juice. The amount of AA obtained with the OECTs is in good agreement with that determined by HPLC and with values reported in the literature for orange juices. Furthermore, these OECTs can be considered promising candidates for the selective detection of AA in the presence of other interfering antioxidants.

\section{Acknowledgments}

This work was supported by Spanish Government / FEDER funds (grant number MAT201564139-C4-3-R (MINECO/FEDER)) and Generalitat Valenciana funds (grant number $\mathrm{AICO} / 2015 / 103)$.

\section{References}

[1] A. Malinauskas, R. Garjonyte, R. Mazeikiene, I. Jureviciute, Electrochemical response of ascorbic acid at conducting and electrogenerated polymer modified electrodes for electroanalytical applications: a review, Talanta 64 (2004) 121-129.

[2] A.M. Pisoschi, A. Pop, A.I. Serban, C. Fafaneata, Electrochemical methods for ascorbic acid determination, Electrochim. Acta 121 (2014) 443-460.

[3] J. Fenoll, A. Martínez, P. Hellín, P. Flores, Simultaneous determination of ascorbic acid and dehydroascorbic acids in vegetables and fruits by liquid chromatography with tandem-mass spectrometry, Food Chem. 127 (2011) 340-344.

[4] T.N. Shekhovtsova, S.V. Muginova, J.A. Luchinina, A.Z. Galimova, Enzimatic methods in food analysis: determination of ascorbic acid, Anal. Chim. Acta 573-574 (2006) 125-132. 
[5] M. Szultka, M. Buszewska-Forajta, R. Kaliszan, B. Buszewski, Determination of ascorbic acid and its degradation products by High-Performance Liquid Chromatography-triple quadrople Mass Spectrometry, Electrophoresis 35 (2014) 585592.

[6] K.K. Chebrolu, G.K. Jayaprakasha, K.S. Yoo, J.L. Jifon, B.S Patil, An improved sample method for quantification of ascorbic acid and dehydroascorbic acid by HPLC, LWT Food Sci. Technol. 47 (2012) 443-449.

[7] L. Novakova, P. Solich, D. Solichova, HPLC methods for simultaneous determination of ascorbic and dehydroascorbic acids, Trends Anal. Chem. 27 (2008) 942-958.

[8] M. Özyürek, K. Güclü, B. Bektasoglu, R. Apak, Spectrophotometric determination of ascorbic acid by the modified CUPRAC method with extractive separation of flavonoids-La(III) complexes, Anal. Chim. Acta 588 (2007) 88-95.

[9] Y.P. Dong, T.T. Gao, X.F. Chu, J. Chen, C.M. Wang, Flow injectionchemiluminescence determination of ascorbic based on luminol-ferricyanide-gold nanoparticles system, J. Lumin. 154 (2014) 350-355.

[10] L. Galiana-Balaguer, S. Roselló, J.M. Herrero-Martínez, A. Maquieira, F. Nuez, Determination of L-ascorbic in Lycopersicon fruits by Capillary Zone Electrophoresis, Anal. Biochem. 296 (2001) 218-224.

[11] L. Suntornsuk, W. Gritsanapun, S. Nilkamhank, A. Paochom, Quantification of vitamin C conten in herbal juice using direct titration, J. Pharm. Biomed. Anal. 28 (2002) 849855.

[12] P. Lin, F. Yan, Organic Thin-Film Transistors for chemical and biological sensing, Adv. Mat. 24 (2012) 34-51.

[13] J.T. Mabeck, G.G. Malliaras, Chemical and biological sensors based on organic thinfilm transistors, Anal. Bioanal. Chem. 384 (2006) 343-353.

[14] L.B. Groenendaal, F. Jonas, D. Freitag, H. Pielartzik, J.R. Reynolds, Poly (3, 4ethylenedioxythiophene) and its derivatives: past, present, and future, Adv. Mat. 12 (2000) 481-494.

[15] S. Kirchmeyer, K. Reuter, Scientific importance, properties and growing applications of poly(3,4-ethylenedioxythiophene), J. Mat. Chem. 15 (2005) 2077-2088.

[16] G. Tarabella, G. Nanda, M. Villani, N. Coppedè, R. Mosca, G.G. Malliaras, C. Santato, S. Iannotta, F. Cicoira, Organic electrochemical transistors for monitoring micelle formation, Chem. Sci. 3 (2012) 3432-3435.

[17] D. Khodagholy, J. Rivnay, M. Sessolo, M. Gurfinkel, P. Leleux, L.H. Jimison, E. Stavrinidou, T. Herve, S. Sanaur, R.M. Owens, G.G. Malliaras, High transconductance organic electrochemical transistors, Nat. Commun. 4 (2013) 2133. 
[18] D. Nilsson, T. Kugler, P.O. Sevensson, M. Berggren, An all-organic sensor-transistor based on a novel electrochemical transducer concept printed electrochemical sensors on paper, Sens. Actuators B 86 (2002) 193-197.

[19] L. Basiricò, P. Cosseddu, A. Scidà, B. Fraboni, G.G. Malliaras, A. Bonfiglio, Electrical characteristics of ink-jet printed all-polymer electrochemical transistors, Org. Electron. 13 (2012) 244-248.

[20] I. Gualandi, M. Marzocchi, A. Achilli, A. Bonfiglio, B. Fraboni, Textile organic electrochemical transistors as a platform for wearable biosensors, Sci. Rep. 6 (2016) 33637.

[21] L. Contat-Rodrigo, C. Pérez-Fuster, J.V. Lidon-Roger, E. GarcíaBreijo, Characterization of screen-printed organic electrochemical transistors to detect cations of different sizes, Sensors 16 (2016) 1599.

[22] P.A. Ersman, d. Nilsson, J. Kawahara, G. Gustafsson, M. Berggren, Fast-switching allprinted organic electrochemical transistors, Org. Electron. 14 (2013) 1276-1280.

[23] D.A. Bernards, G.G. Malliaras, Steady-state and transient behavior of organic electrochemical transistors, Adv. Funct. Mat. 17 (2007) 3538-3544.

[24] M. Nikolou, G.G. Malliaras, Applications of poly (3, 4-ethylenedioxythiophene) doped with poly (styrene sulfonic acid) transistors in chemical and biological sensors, Chem. Rec. 8 (2008) 13-22.

[25] D. Nilsson, N. Robinson, M. Berggren, R. Forchheimer, Electrochemical logic circuits, Adv. Mat. 17 (2005) 353-358.

[26] P. Lin, F. Yang, H.L.W. Chan, Ion-sensitive properties of organic electrochemical transistors, Appl. Mat. Interfaces 2 (2010) 1637-1641.

[27] G. Tarabella, C. Santato, S. Y. Yang, S. Iannotta, G.G. Malliaras, S. Cicoira, Effect of the gate electrode on the response of organic electrochemical transistors, Appl. Phys. Lett. 97 (2010) 123304.

[28] F. Cicoira, M. Sessolo, O. Yaghmazadeh, J.A. DeFranco, S.Y. Yang, G.G. Malliaras, Influence of device geometry on sensor characteristics of planar organic electrochemical transistors, Adv. Mat. 22 (2010) 1012-1016.

[29] O. Yaghmazadeh, F. Cicoira, D.A. Bernards, S.Y. Yang, Y. Bonnassieux, G.G. Malliaras, Optimization of organic electrochemical transistors for sensor applications, J. Polym. Sci. B Polym. Phys. 49 (2011) 34-39.

[30] I. Gualandi, M. Marzocchi, E. Scavetta, M. Calienni, A. Bonfiglio, B. Fraboni, A simple all-PEDOT:PSS electrochemical transistor for ascorbic acid sensing, J. Mater. Chem. B 3 (2015) 6753-6762.

[31] C. Zanardi, F. Terzi, R. Seeber, Polythiophenes and polythiophene-based composites in amperometric sensing, Anal. Bional. Chem. 405 (2013) 509-531. 
[32] G. Xu, D. Liu, J. Chen, X. Ye, Y. Ma, J. Shi, Juice components and antioxidant capacity of citrus varieties cultivated in China, Food Chem. 106 (2008) 545-551.

[33] R. Re, N. Pellegrini, A. Proteggente, A. Pannala, M. Yang, C. Rice-Evans, Antioxidant activity applying an improved ABTS radical cation decolorization assay, Free Radic. Biol. Med. 26 (1999) 1231-1237.

[34] F. Lin, M.C. Lonergan, Gate electrode processes in an electrolyte-gated transistor: nonFaradaically versus Faradaiclly coupled conductivity modulation of a polyacetylene ionomer, App. Phys. Lett. 88 (2006) 133507.

[35] D.A. Bernards, D.J. Macaya, M. Nikolou, J.A. DeFranco, S. Takamatsu, G.G. Malliaras, Enzimatic sensing with organic electrochemical transitors, J. Mater. Chem. 18 (2008) 116-120.

[36] P.J. O‘Connell, C. Gormally, M. Pravda, G.G. Guilbault G.G. Development of an amperometric L-ascorbic acid (Vitamin C) sensor based on electropolymerised aniline for pharmaceutical and food analysis, Anal. Chim. Acta 431 (2001) 239-247.

[37] P. Rapisarda, A. Tomaino, R. Lo Cascio, F. Bonina, A. De Pasquale, A. Saija, Antioxidant effectiveness as influenced by phenolic content of fresh orange juices, $\mathrm{J}$. Agric. Food Chem. 47 (1999) 4718-4723. 


\section{Screen-Printed Organic Electrochemical Transistors for the Detection of Ascorbic Acid in Food}

- The use of screen-printed all-PEDOT:PSS Organic Electrochemical Transistors (OECTs) for the determination of ascorbic acid (AA) in food is reported.

- The effect of the geometry on the transistors performance was investigated by comparing the response of OECTs with different channel and gate areas ratio $(\gamma)$.

- OECTs with large $\gamma$ show the best performance for AA sensing. These devices exhibit smaller background, higher sensitivity, larger modulation and better limit of detection.

- The reliability of the proposed sensors in real food samples was demonstrated by measuring the OECTs response in a commercial orange juice.

- These OECTs have shown to be sensitive enough for quantitatively monitoring AA in food, and to be promising candidates for selective AA detection in the presence of other interfering antioxidants. 